\title{
Precluding oil leakage from the oil ship tankers under mechanical damage
}

\begin{abstract}
The problem of Protection of sea medium with the help of low temperature engineering is discussed. The special item is the way how to preclude oil leakage which is shipped with sea tankers in case of mechanical damage of it. It is suggested to set up vertical flexible tubs which are fed by cryogenic fluids when the mechanical damage of the ship hull occurred. Water ices are forming on the tube surface and preclude oil leakage from the tanks.

The heat transfer task under formation of water ice is considered. Fourier equation for a layer of water ice forming on a cylindrical tube wall being at low temperature surrounded by a water medium was solved with a temperature dependence ice heat conductivity et it.
\end{abstract}

Keywords: water ice, fourier unstionale differential equation, temperature dependence of heat conductivity of water ice, heat transfer task
Volume 3 Issue 3 - 2018

\author{
Marinyuk Boris, Ugolnikova Maria \\ Department of Low Temperature Engineering, Moscow State \\ University of Mechanical Engineering (MAMI), Russia
}

\begin{abstract}
Correspondence: Marinyuk Boris, Department of Low Temperature Engineering named by PL Kapitsa, Moscow State University of Mechanical Engineering (MAMI), Moscow, Russia, Tel 8916-9263-952, Email marinykB@yandex.ru
\end{abstract}

Received: April 16, 2018| Published: June 27, 2018

\section{Introduction}

Preservation and transportation of bulk quantities of oil products in tanks is strictly connected with a creation of safety conditions under its operation. Oil leakage in to environment medium may cause a detriment to it. This is equally attributed to the case when the tank is located on the dry land or at the transportation ships. It is known, that cost of water purification from the oil is reached more than $10^{6} \$$ if the sea surface is one mile sq. The damage of tanks wall is possible when the tankers are collided with underwater obstacles, icebergs or other ships. Now in case of such an emergency situation with the tankers, an auxiliary vessel with empty reservoir is called and oil product is pump out from the damaged tank. The span of arrayal time depends on geographical point of the incident and takes from one to a few days. During this time pollution of sea may spared to great scales. There is no garantec of complete protection from oil leakage if the tanker has double hull. Low temperature engineering technology gives a method which may solve the problem of precluding of oil leakage from the damaged wall of ship tanks with en a short span of time .It is necessary to equip each tank-bearer with a cryogenic reservoir containing liquid nitrogen. The outer side of each tank is supplied by flexible vertical tubs which are set up in parallel with interval of $300 \mathrm{~mm}$. The free hanging of the tubs is preventing them from mechanical damage in case of sea incident. So let it consider the process of water freezing around a tube which is cooled down inside by a liquid nitrogen. To simplify the analysis the temperature of wall tube is accepted as constant at the level of $77 \mathrm{~K}$ (boiling temperature of liquid nitrogen at atmospheric pressure).

The heat transfer coefficient from the water side is constant $\alpha \mathrm{w}=$ $180 \mathrm{Wt} /\left(\mathrm{m}^{2} \mathrm{~K}\right)$.

The heat transfer process is described by Fourier unstationary differential equation for a tube being cooled inside and immersed in an aqueous medium.

In cylindrical coordinates it looks like this

$$
c_{p} \rho \frac{d T}{d \tau}=\frac{d}{d r}\left[\lambda(T) \frac{d T}{d r}\right]+\frac{\lambda}{r} \frac{d T}{d r}
$$

Equation (1) is attributed to unlinear unstationary type with variable transfer coefficient, which can't be solved in quadrature by methods. More fertile results may be obtained on the bases of approximate analytical methods which give result with an desirable accuracy where

$c_{p}$ - is average specific heat accumulation of ice at a temperature

$$
\bar{T}=\frac{T_{p h}-T_{\text {wall }}}{2}
$$

$T_{\text {wal }}$ - Temperature of phase change of water in to ice, $\mathrm{K}$;

$T_{\text {wall }}$ - Temperature of wall of tube, $\mathrm{K}$;

The law of heat conductivity change of ice with temperature is correlated by K.D. Timmerhaus et al. ${ }^{1}$

$$
\lambda=\frac{K}{T}
$$

Where $\mathrm{K}=615.34 \mathrm{~W} / \mathrm{m}$ - dimensional constant;

T-Temperature of ice, $\mathrm{K}$

$\lambda_{0}$-heat conductivity of water ice at phase change temperature, $\mathrm{W} /(\mathrm{m} \mathrm{K})$;

$\rho$-Is density, $\mathrm{kg} / \mathrm{m}^{3} ;$
$r$ - Cylindrical coordinate, $\mathrm{m}$; 
$\tau$-Time of process, $\mathrm{s}$.

An initial and bordering conditions is expressed as

$$
\begin{aligned}
& T(r, 0)=T_{w} \\
& T(r, 0)=T_{w} \\
& T(\eta, \tau)=T_{p h}
\end{aligned}
$$

Where $T_{w}$ temperature of tube wall, $\mathrm{K}$;

$\eta$-Movable coordinates of ice water border, $\mathrm{m}$;

$T_{w}$-Water temperature, $\mathrm{K}$.

Heat balance on the movable ice water border

$$
\left.\lambda_{0} \frac{d T}{d r}\right|_{r=\eta}=\alpha_{w}\left(T_{w}-T_{p h}\right)+\rho L \frac{d \eta}{d r}
$$

Where $L$-heat of phase changes (freezing of water into ice), $\mathrm{Dj} / \mathrm{kg}$.

The final result of the equation (1) decision without deriving of it is presented at. ${ }^{2}$ The procedure of deriving of the result provides an introduction of new variable "V"

$$
\begin{aligned}
& V=\frac{r-r_{0}}{\sqrt{\tau}} \\
& T(r, \tau)=T(V) \\
& (\eta-)=(\tau)=\beta \sqrt{\tau}
\end{aligned}
$$

Where $\xi$-outside of the tube, m;

$\xi$-Thickness of ice layer forming on the tube surface, m;

$\beta$-Variable factor office growth rate, $\mathrm{m} / \mathrm{c}^{2}$. them

This turning the equation (1) from partial derivation to a full of

$$
c \rho \frac{d T}{d V}=\frac{d}{d V}\left[\frac{K}{T} \frac{d T}{d V}\right]+\frac{1}{V \sqrt{\tau}+r_{0}} \frac{K}{T} \frac{d T}{d V}
$$

Equation (6) is taking the form

$$
\left.\frac{\lambda}{\sqrt{\tau}} \frac{d T}{d V}\right|_{V=\beta}=\rho L \frac{\beta}{2 \sqrt{\tau}}+\alpha_{w}\left(T_{w}-T_{p h}\right)
$$

Initial and bordering conditions will be

$$
\begin{aligned}
& T(\infty)=T_{w} \\
& T(\infty)=T_{w} \\
& T(\beta)=T_{p h}
\end{aligned}
$$

Suppose the temperature profiles within the ice lager is expressed as a series

$T(V)=T(\beta)+\frac{T^{\prime}(\beta)}{1 !}(V-\beta)+\frac{T^{n}(\beta)}{2 !}(V-\beta)^{2}+\ldots+\frac{T^{n}(\beta)}{n !}(V-\beta)^{n}$

The second derivative of $\mathrm{T}(\mathrm{V})$

$$
T^{\prime \prime}=\frac{\left(T^{\prime}\right)^{2}}{(16 \& 73}-\frac{T^{\prime} \sqrt{\tau}}{\beta \sqrt{\tau}+r_{0}}+\frac{273 c \rho T^{\prime}}{2 K}
$$

Taking into account expressions $(11,12,13$, and 14) the final result of the task is expressed.

$$
T_{\text {wall }}=T_{p h}-\left[\frac{\rho L \beta}{2 \lambda}+\frac{\alpha_{w} \sqrt{\tau}}{\lambda}\left(T_{w}-T_{p h}\right)\right] \beta++\left[\begin{array}{l}
\frac{\left(\frac{\rho L \beta}{2 \lambda}+\frac{\alpha_{w} \sqrt{\tau}}{\lambda}\left(T_{w}-T_{p h}\right)\right)^{2}}{T_{p h}}-\frac{\left(\frac{\rho L \beta}{2 \lambda}+\frac{\alpha_{w} \sqrt{\tau}}{\lambda}\left(T_{w}-T_{p h}\right)\right) \sqrt{\tau}}{\beta \sqrt{\tau}+r_{0}} \\
+\frac{\rho c_{p} \beta T_{p h}}{2 K}\left[\frac{\rho L \beta}{2 \lambda}+\frac{\alpha_{w} \sqrt{\tau}}{\lambda}\left(T_{w}-T_{p h}\right)\right] \frac{\beta^{2}}{2}
\end{array}\right] \frac{\beta^{2}}{2}
$$

Variable factor of growth rate $\beta$ is found for a accepted time by solving (17). The ice thickness is detcrmed by equation (9). The calculation made according equation tube (17) and (9) revealed that cryogenic temperatures on the wall needs taking into account the temperature dependence of water ice layer (Figure 1).

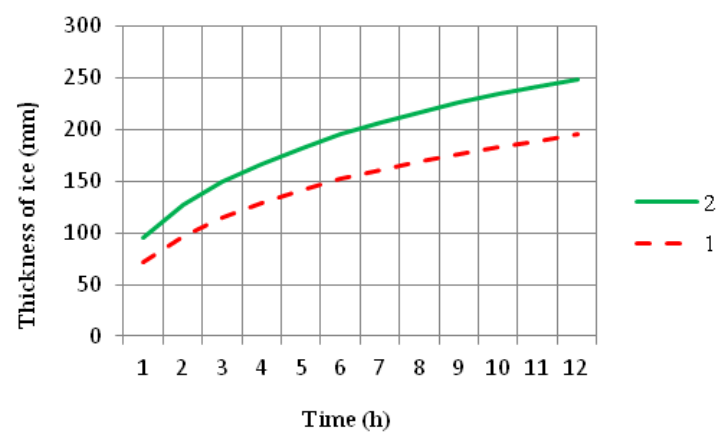

Figure I Growth of ice on a flat wall being at cryogenic temperatures and immersed in aqua media $\left(T_{w}=278 \mathrm{~K}, \alpha_{w}=180 \mathrm{~W} /\left(\mathrm{m}^{2} \mathrm{~K}\right), \mathrm{T}_{\text {wall }}=77 \mathrm{~K}\right.$, radius of the tube $r=0.04 \mathrm{~m}$ ): I - with constant characteristics of ice; 2 - with variable characteristics of ice.

\section{Conclusion}

1. The problem of Protection of sea medium with the help of low temperature engineering is discussed.

2. It is suggested to set up vertical flexible tubs with cryogenic liquid nitrogen on the surface of the tanks. 
3. Fourier equation for a layer of water ice cylinder being cooled from inside and surrounded by a water medium was solved with a temperature dependence of ice heat conductivity.

\section{Acknowledgements}

None.

\section{Conflict of interest}

The author declares there is no conflict of interest.

\section{References}

1. Dean JW, Timmerhaus KD. Thermal Conductivity Of solid $\mathrm{O}$ and $\mathrm{O}$ at Low Temperatures. Adv in Cryogenic Engineering. 1963;8:63-67.

2. Marinyuk BT, Ugolnikova MA. The Dynamics of ice Formation on a tubs elements ice generators. $J$ of Refrigeration. 2016;12:44-47. 